\title{
Stabilité $L^{p}$ exponentielle d'un système d'échangeurs thermiques avec diffusion et sans diffusion
}

\author{
Abdoua Tchousso ${ }^{\mathrm{a}}-\mathrm{Xiao-Dong} \mathrm{Li}^{\mathrm{b}}-$ Cheng-Zhong $\mathrm{Xu}^{\mathrm{b}}{ }^{*}{ }^{*}-$ Gauthier \\ Sallet ${ }^{\mathrm{c}}$ \\ a Département de Mathématiques et Informatique \\ Université Abdou Moumouni de Niamey, BP 10662 Niamey - Niger \\ tchousso@yahoo.fr \\ b Université de Lyon, F-69622, Lyon, France - Université Lyon 1, Villeurbanne \\ CNRS, UMR5007, Laboratoire d'Automatique et de Génie des Procédés \\ lix@lagep.cpe.fr — * Correspondind author : xu@lagep.univ-lyon1.fr \\ *** Laboratoire et Département de Mathématiques, CNRS, UMR 7122 \\ Université de Metz, Bâtiment A, Île du Saulcy, F-57045 Metz Cedex 01 \\ sallet@loria.fr
}

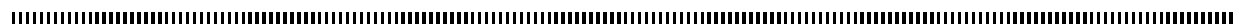

ABSTRACT. In this paper we study exponential stability of a heat exchanger system with diffusion and without diffusion in the context of Banach spaces. The heat exchanger system is governed by hyperbolic partial differential equations (PDE) and parabolic PDEs, respectively, according to the diffusion impact ignored or not in the heat exchange. The exponential stability of the model with diffusion in the Banach space $(C[0,1])^{4}$ is deduced by establishing the exponential $L^{p}$ stability of the considered system, and using the sectorial operator theory. The exponential decay rate of stability is also computed for the model with diffusion. Using the perturbation theory, we establish the exponential stability of the model without diffusion in the Banach space $(C[0,1])^{4}$ with the uniform topology. However the exponential decay rate of stability without diffusion is not exactly computed, since its associated semigroup is non analytic.

Indeed the purpose of our paper is to investigate the exponential stability of a heat exchanger system with diffusion and without diffusion in the real Banach space $X_{\infty}=(C[0,1])^{4}$ with the uniform norm. The exponential stability of these two models in the Hilbert space $X_{2}=\left(L^{2}(0,1)\right)^{4}$ has been proved in [31] by using Lyapunov's direct method. The first step consists to study the stability problem in the real Banach space $X_{p}=\left(L^{p}(0,1)\right)^{4}$ equipped with the usual $L^{p}$ norm, $p>1$. By passing to the limit $(p \rightarrow \infty)$ we can extend some results of exponential stability from $X_{p}=\left(L^{p}(0,1)\right)^{4}$ to the space $X_{\infty}=(C[0,1])^{4}$. In particular the dissipativity of the system in all the $X_{p}$ spaces implies its dissipativity in $X_{\infty}$ (see Lemma 3).

The section 1 is dedicated to recall the heat exchanger models. The process with diffusion is governed by a system of parabolic PDEs, and the process without diffusion is described by degenerate hyperbolic PDEs of first order. The section 2 deals with exponential stability of the parabolic system in the Lebesgue spaces $L^{p}(0,1), 1<p<\infty$. Certain results can be extended to the $X_{\infty}$ space. Unfortunately this study doesn't allow us to deduce the expected stability of the system in $X_{\infty}$. In the section 3 , the sectorial operator theory is made use of to get exponential stability results on the model with diffusion in $X_{p}$. Specifically the theory enables us to determine the exponential decay rate in $(C[0,1])^{4}$ by computing the spectrum bound. In the section 4 , using a perturbation technique we show the exponential stability for the model without diffusion in all $X_{p}$ spaces, $1<p<\infty$. We then 
take the limit, as $p$ goes to $\infty$, to deduce the exponential stability of the system in the Banach space $X_{\infty}$.

We call the diffusion model the heat exchanger model with diffusion taken into account and the convection model the heat exchanger without diffusion, respectively. We use the analyticity property of the semigroup associated to the diffusion model in order to determine its exponential decay rate. However the semigroup associated to the convection model is not analytic. In the latter case we have not yet found an efficient method to compute exactly the exponential decay rate.

The main tools we use for our investigations are the notion of dissipativity in the Banach spaces, specifically in the $L^{p}$ spaces, and the sectorial operator theory. As the reader will see our work presents some extensions of the Lyapunov's direct method to a context of Banach spaces.

We will denote the system operator associated to the diffusion model by $A_{d, p}$, and that of the convection model by $A_{c, p}$, respectively. The index $p$ indicates the $L^{p}(\Omega)$ space in which the system evolves and the operator $A_{d, p}$ or $A_{c, p}$ is considered. Thus $A_{d, p}$ (resp. $A_{c, p}$ ) indicates the diffusive (resp. convective) operator in the $X_{p}$ space.

RÉSUMÉ. L'objectif de cet article est d'étudier la stabilité exponentielle des systèmes d'échangeurs thermiques, respectivement, avec diffusion et sans diffusion, dans le cadre de l'espace de Banach réel $X_{\infty}=(C[0,1])^{4}$ muni de la norme uniforme. La stabilité exponentielle de ces deux modèles dans l'espace de Hilbert $X_{2}=\left(L^{2}(0,1)\right)^{4}$ a été établie dans [31] en utilisant la méthode de Lyapunov directe.

La démarche entreprise ici consiste à étudier le problème de la stabilité dans les espaces de Banach réels $X_{p}=\left(L^{p}(0,1)\right)^{4}$ muni de la norme $L^{p}$ avec $p>1$. Par passage à la limite $(p \rightarrow+\infty)$ on peut dans certains cas étendre les résultats de stabilité exponentielle de $X_{p}=\left(L^{p}(0,1)\right)^{4}$ à l'espace $X_{\infty}=(C[0,1])^{4}$. En effet la dissipativité du système étudié dans tous les espaces $X_{p}$ entraîne sa dissipativité dans $X_{\infty}$ (voir le Lemme 3 ).

La première section est consacrée au rappel des modèles des échangeurs thermiques. Le processus avec diffusion se modélise par un système d'équations aux dérivées partielles du type parabolique, tandis que le processus sans diffusion est décrit par un système hyperbolique du premier ordre. La deuxième section traite de la stabilité exponentielle du système parabolique dans le cadre des espaces $L^{p}(0,1), 1<p<\infty$. On en déduit des résultats pour l'espace $X_{\infty}$. Néanmoins cette étude ne permet pas de déduire la stabilité du système dans $X_{\infty}$.

Les résultats de stabilité exponentielle dans $X_{p}$ pour le modèle avec diffusion sont établis dans la troisième section en utilisant la théorie des opérateurs sectoriels. Mieux, cette théorie permet de prouver la stabilité exponentielle dans l'espace $\left(C^{1}[0,1]\right)^{4}$. Dans la quatrième section, en utilisant un résultat de perturbation on démontre la stabilité exponentielle pour le modèle sans diffusion dans tous les espaces $X_{p}, 1<p<\infty$. En utilisant le passage à la limite évoqué plus haut, on déduit la stabilité exponentielle du système dans le Banach $X_{\infty}$.

KEYWORDS : Partial differential equations, exponential stability, Lyapunov's direct method, semigroup theory

MOTS-CLÉS : Équations aux dérivées partielles, stabilité exponentielle, Méthode de Lyapunov directe, théorie du semi-groupe 


\section{Introduction}

L'objectif de cet article est d'étudier la stabilité exponentielle des systèmes d'échangeurs thermiques, respectivement, avec diffusion et sans diffusion, dans le cadre de l'espace de Banach réel $X_{\infty}=(C[0,1])^{4}$ muni de la norme uniforme. La stabilité exponentielle de ces deux modèles dans l'espace de Hilbert $X_{2}=\left(L^{2}(0,1)\right)^{4}$ a été établie dans [31] en utilisant la méthode de Lyapunov directe.

La démarche entreprise ici consiste à étudier le problème de la stabilité dans les espaces de Banach réels $X_{p}=\left(L^{p}(0,1)\right)^{4}$ muni de la norme $L^{p}$ avec $p>1$. Par passage à la limite $(p \rightarrow+\infty)$ on peut dans certains cas étendre les résultats de stabilité exponentielle de $X_{p}=\left(L^{p}(0,1)\right)^{4}$ à l'espace $X_{\infty}=(C[0,1])^{4}$. En effet la dissipativité du système étudié dans tous les espaces $X_{p}$ entraîne sa dissipativité dans $X_{\infty}$ (voir le Lemme 3).

La première section est consacrée au rappel des modèles des échangeurs. Le processus avec diffusion se modélise par un système d'équations aux dérivées partielles du type parabolique, tandis que le processus sans diffusion est un système hyperbolique d'ordre un du type dégénéré. La deuxième section traite de la stabilité exponentielle du système parabolique dans le cadre des espaces $L^{p}(0,1), 1<p<\infty$. On en déduit des résultats pour l'espace $X_{\infty}$. Néanmoins cette étude ne permet pas de déduire la stabilité du système dans $X_{\infty}$.

Les résultats de stabilité exponentielle dans $X_{p}$ pour le modèle avec diffusion sont établis dans la troisième section en utilisant la théorie des opérateurs sectoriels. Mieux, cette théorie permet de prouver la stabilité exponentielle dans l'espace $\left(C^{1}[0,1]\right)^{4}$.

Dans la quatrième section, en utilisant un résultat de perturbation on démontre la stabilité exponentielle pour le modèle sans diffusion dans tous les espaces $X_{p}, 1<p<\infty$. On utilise alors le passage à la limite évoqué plus haut pour déduire la stabilité exponentielle du système dans le Banach $X_{\infty}$.

Dans la suite on appelle modèle de diffusion le modèle d'échangeurs thermiques avec diffusion et, de même, modèle de convection le modèle d'échangeurs thermiques sans diffusion. Nous nous servirons de l'analyticité du semi-groupe associé au modèle de diffusion pour estimer son taux de décroissance exponentielle. Or le semi-groupe associé au modèle de convection n'est pas analytique. Pour ce dernier cas nous n'avons pas encore trouvé de méthode efficace permettant de déterminer exactement le taux de décroissance exponentielle.

Les principaux outils que l'on utilise pour cette étude sont la notion de la dissipativité dans les espaces de Banach, plus particulièrement dans les espaces $L^{p}$, et la théorie des opérateurs sectoriels. Cet article présente une extension de la méthode de Lyapunov directe pour un contexte des espaces de Banach.

Dans la suite on notera par $A_{d, p}$ l'opérateur associé au modèle de diffusion, et par $A_{c, p}$ celui de convection, respectivement. L'indice $p$ indique l'espace $L^{p}(\Omega)$ dans lequel le système évolue et l'opérateur $A_{d, p}$ est étudié. Ainsi $A_{d, p}$ (resp. $A_{c, p}$ ) désignera l'opérateur diffusive (resp. convective) dans l'espace $X_{p}$. 


\section{Présentation des modèles}

Le dispositif du réseau d'échangeurs thermiques que l'on étudie est présenté à la Figure 1,
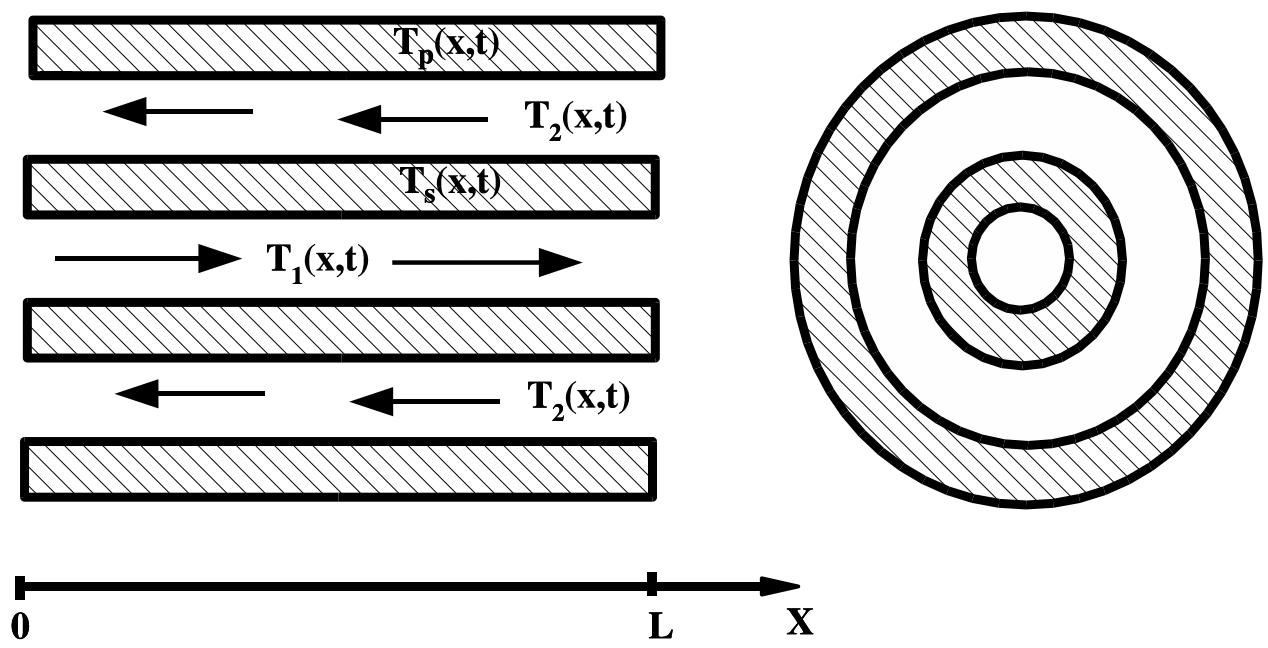

Figure 1. réseau d'échangeurs thermiques

où

- $T_{1}$ est la température du fluide à chauffer,

- $T_{2}$ est la température du fluide chauffant,

- $T_{p}$ est la température de la paroi isolatrice,

- $T_{s}$ est la température de la paroi séparatrice.

Il s'agit d'un dispositif d'échange de chaleur constitué de deux cylindres coaxiaux séparés entre eux par la paroi du milieu et isolés de l'extérieur par la seconde parois. Deux fluides de caractéristiques différentes circulent à contre-courant dans les cylindres. Ces fluides échangent de la chaleur à travers la paroi du milieu. Il existe deux modèles permettant de décrire ce procédé :

- Le premier modèle qui tient compte de la diffusion de la chaleur dans les différents corps, i.e., le modèle avec diffusion. Dans ce cas le modèle est du type parabolique (appelé modèle de diffusion).

- Le second modèle dans lequel la diffusion de la chaleur est négligée par rapport au phénomène du transport (de la convection). On obtient le modèle sans diffusion (appelé aussi modèle de convection). Dans ce dernier cas le modèle est décrit par des équations aux dérivées partielles (EDP en abrégé) du type hyperbolique d'ordre un, mais dégénéré. 
Pour plus de détails sur la modélisation des échangeurs thermiques, on peut se rapporter à [31] et à la littérature citée dans [31].

On pose $u_{1}=T_{1}, u_{2}=T_{s}, u_{3}=T_{2}, u_{4}=T_{p}$. Le système d'échangeurs diffusives se modélise comme suit:

$$
\left(\Sigma_{d}\right):\left\{\begin{array}{l}
\partial_{t} u_{1}=\alpha_{1} \partial_{x x} u_{1}-F_{1} \partial_{x} u_{1}+h_{1}\left(u_{2}-u_{1}\right), \quad \forall(x, t) \in(0,1) \times(0, \infty) \\
\partial_{t} u_{2}=\alpha_{2} \partial_{x x} u_{2}+h_{2}\left(u_{1}-u_{2}\right)+h_{3}\left(u_{3}-u_{2}\right) \\
\partial_{t} u_{3}=\alpha_{3} \partial_{x x} u_{3}+F_{2} \partial_{x} u_{3}+h_{4}\left(u_{2}-u_{3}\right)+h_{5}\left(u_{4}-u_{3}\right) \\
\partial_{t} u_{4}=\alpha_{4} \partial_{x x} u_{4}+h_{6}\left(u_{3}-u_{4}\right) \\
u_{1}(0, t)=0, \partial_{x} u_{1}(1, t)=0 \\
\partial_{x} u_{2}(0, t)=0, \partial_{x} u_{2}(1, t)=0 \\
\partial_{x} u_{3}(0, t)=0, u_{3}(1, t)=0 \\
\partial_{x} u_{4}(0, t)=0, \partial_{x} u_{4}(1, t)=0 \\
u(x, 0)=u^{0}(x), \quad \forall x \in(0,1)
\end{array}\right.
$$

où $\alpha_{i}, i=1, \ldots, 4, h_{j}, j=1, \ldots, 6$, et $F_{k}, k=1,2$, sont des constantes positives. Les EDP $\left(\Sigma_{d}\right)$ permettent de décrire la dynamique des variations de températures par rapport au point d'équilibre défini par les températures constantes d'entrée à l'échangeur des fluides. Les gradients nuls à la frontière expriment le fait que le flux de chaleur vers l'extérieur est nul.

Le modèle de convection est gouverné par les EDP hyperboliques suivantes :

$$
\left(\Sigma_{c}\right):\left\{\begin{array}{l}
\partial_{t} u_{1}=-F_{1} \partial_{x} u_{1}+h_{1}\left(u_{2}-u_{1}\right), \quad \forall(x, t) \in(0,1) \times(0, \infty) \\
\partial_{t} u_{2}=h_{2}\left(u_{1}-u_{2}\right)+h_{3}\left(u_{3}-u_{2}\right) \\
\partial_{t} u_{3}=F_{2} \partial_{x} u_{3}+h_{4}\left(u_{2}-u_{3}\right)+h_{5}\left(u_{4}-u_{3}\right) \\
\partial_{t} u_{4}=h_{6}\left(u_{3}-u_{4}\right) \\
u_{1}(0, t)=0, u_{3}(1, t)=0 \\
u(x, 0)=u^{0}(x), \quad x \in(0,1)
\end{array}\right.
$$

De même que dans le modèle de diffusion, les constantes sont réelles et positives.

\section{Stabilité du modèle avec diffusion dans $X_{p}=\left(L^{p}(0,1)\right)^{4}$}

\subsection{Rappels de la dissipativité dans les espaces $L^{p}$}

Considérons l'espace de Banach réel $L^{p}(0,1)$, avec $p \in(1,+\infty)$. Les propriétés suivantes sont classiques:

$-L^{p}(0,1)$ est un espace réflexif et séparable.

- Le dual de $L^{p}(0,1)$ est identifié à $L^{q}(0,1)$ (via un isomorphisme de Banach), avec $q=\frac{p}{p-1} \in(1,+\infty)$.

- Le produit $<\cdot, \cdot>_{q, p}$ de la dualité sur $L^{p} \times L^{q}$ s'écrit:

$$
\forall f \in L^{p}(0,1), g \in L^{q}(0,1) ; \quad<g, f>_{q, p}=\int_{0}^{1} g(x) f(x) d x .
$$


Pour les espaces produits $X_{p}=\left(L^{p}(0,1)\right)^{n}, p \in(1,+\infty)$ et $n \geq 1$, les propriétés similaires sont vraies :

$-X_{p}$ est un espace de Banach pour la norme suivante

$$
\forall f \in X_{p}, \quad\|f\|_{p}=\left(\sum_{i=1}^{n}\left\|f_{i}\right\|_{p}^{2}\right)^{\frac{1}{2}}
$$

où $\left\|f_{i}\right\|_{p}=\left(\int_{0}^{1}\left|f_{i}(x)\right|^{p}\right)^{\frac{1}{p}}$.

$-X_{p}$ est un espace Banach réflexif et séparable.

- Le dual topologique de $\left(L^{p}(0,1)\right)^{n}$ est identifié à $\left(L^{q}(0,1)\right)^{n}$ (via un isomorphisme de Banach), avec $q=\frac{p}{p-1} \in(1,+\infty)$.

- Le produit de la dualité sur $X_{p} \times X_{q}$ s'écrit:

$$
\forall f \in X_{p}, \forall g \in X_{q}, \quad<g, f>_{q, p}=\int_{0}^{1} g(x) \cdot f(x) d x=\sum_{i=1}^{n} \int_{0}^{1} g_{i}(x) f_{i}(x) d x .
$$

La notion de la dissipativité d'un opérateur linéaire $A$ dans un Hilbert $H$ est bien connue. $A$ est dissipatif si et seulement si $\forall f \in D(A),<A f, f>_{H} \leq 0$. Par contre dans un espace de Banach, il faut avoir recours à la notion d'ensemble de dualité pour définir la dissipativité.

Définition 1. Soit $X$ un espace de Banach et soit $X^{*}$ son dual topologique.

- Pour $f \in X$, l'ensemble de dualité de $f$, noté $J(f)$, est défini par:

$$
J(f)=\left\{g \in X^{*}:\|g\|_{X^{*}}=\|f\|_{X},<g, f>_{X^{*}, X}=\|f\|_{X}^{2}\right\} .
$$

- Un opérateur linéaire A sur X est dit dissipatif si

$$
\forall f \in D(A), \quad \exists g \in J(f) /<g, A f>_{X^{*}, X} \leq 0 .
$$

On a aussi la caractérisation suivante des opérateurs dissipatifs.

Proposition 1. Un opérateur linéaire A sur un Banach X est dissipatif si et seulement $s i$

$$
\forall f \in D(A), \forall \lambda>0, \quad\|(\lambda I-A) f\|_{X} \geq \lambda\|f\|_{X}
$$

Preuve. Voir le Théorème 1-4.2 in [22].

Revenons aux espaces $L^{p}(0,1)$ et $X_{p}=\left(L^{p}(0,1)\right)^{n}, p \in(1,+\infty)$ et $n \geq 1$. Dans le Banach $L^{p}(0,1)$, on caractérise complètement l'ensemble de dualité.

Lemme 1. Soit $f \in L^{p}(0,1)$. Alors l'ensemble de dualité de $f$ dans $L^{p}(0,1)$ est le singleton

$$
J_{p}(f)=\left\{f^{*}=\frac{|f|^{p-2} f}{\|f\|_{p}^{p-2}}\right\} .
$$

Pour le Banach $X_{p}=\left(L^{p}(0,1)\right)^{n}$, l'ensemble de dualité pour chaque élément est aussi un singleton. 
Lemme 2 Soit $f \in X_{p}$. Alors l'ensemble de dualité $J(f)$ de $f$ dans $X_{p}$ est constitué d'un seul élément $g=\left(g_{1}, \ldots, g_{n}\right)^{T}$ défini par

$$
g_{i}=\frac{\left|f_{i}\right|^{p-2} f_{i}}{\left\|f_{i}\right\|_{p}^{p-2}} ; \quad i=1, \ldots, n .
$$

Preuve. Évidemment, $g \in X_{q}$, avec $q=\frac{p}{p-1}$, car

$$
\left\|g_{i}\right\|_{q}=\frac{1}{\left\|f_{i}\right\|_{p}^{p-2}}\left[\int_{0}^{1}\left(\left|f_{i}(x)\right|^{p-1}\right)^{\frac{p}{p-1}}\right]^{\frac{p-1}{p}}=\left\|f_{i}\right\|_{p}<+\infty .
$$

et

$$
\|g\|_{q}=\left(\sum_{i=1}^{n}\left\|g_{i}\right\|_{q}^{2}\right)^{\frac{1}{2}}=\|f\|_{p}
$$

Enfin, on a

$$
<g, f>_{q, p}=\sum_{i=1}^{n} \int_{0}^{1} g_{i}(x) f_{i}(x) d x=\sum_{i=1}^{n} \int_{0}^{1}\left|f_{i}(x)\right|^{p} d x /\left\|f_{i}\right\|_{p}^{p-2}=\|f\|_{p}^{2} .
$$

Donc, $g \in J(f)$. D'autre part, puisque $X_{p}^{*}=X_{q}$ est strictement convexe, $J(f)$ est réduit à un seul élément (voir [6, p.3]).

\subsection{Stabilité exponentielle du modèle avec diffusion dans les espaces $\left(L^{p}(0,1)\right)^{4}$}

Considérons le système $\left(\Sigma_{d}\right)$ d'échangeurs thermiques avec diffusion. On se place dans le Banach $X_{p}=\left(L^{p}(0,1)\right)^{4}$ muni de la norme définie par (1). Associons à ce système l'opérateur dans $X_{p}$ défini par

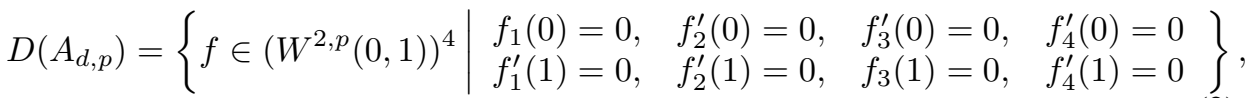

$$
\begin{aligned}
& A_{d, p} f=\left[\begin{array}{l}
\alpha_{1} f_{1}^{\prime \prime}-F_{1} f_{1}^{\prime}+h_{1}\left(f_{2}-f_{1}\right) \\
\alpha_{2} f_{2}^{\prime \prime}+h_{2}\left(f_{1}-f_{2}\right)+h_{3}\left(f_{3}-f_{2}\right) \\
\alpha_{3} f_{3}^{\prime \prime}+F_{2} f_{3}^{\prime}+h_{4}\left(f_{2}-f_{3}\right)+h_{5}\left(f_{4}-f_{3}\right) \\
\alpha_{4} f_{4}^{\prime \prime}+h_{6}\left(f_{3}-f_{4}\right)
\end{array}\right] .
\end{aligned}
$$

Rappelons les espaces de Sobolev $W^{2, p}$ définis par

$$
W^{2, p}(0,1)=\left\{f_{1} \in L^{p}(0,1) \mid f_{1}^{\prime}, f_{1}^{\prime \prime} \in L^{p}(0,1)\right\} .
$$

Effectuons la transformation suivante sur l'opérateur $A_{d, p}$ :

$$
A_{p}=T^{-1} A_{d, p} T
$$

où $T$ est la matrice diagonale d'ordre 4 telle que

$$
T=\operatorname{diag}\left(1, \sqrt{\frac{h_{2}}{h_{1}}}, \sqrt{\frac{h_{2} h_{4}}{h_{1} h_{3}}}, \sqrt{\frac{h_{2} h_{4} h_{6}}{h_{1} h_{3} h_{5}}}\right) .
$$


L'opérateur $A_{p}$ s'écrit alors

$$
\begin{aligned}
& D\left(A_{p}\right)=D\left(A_{d, p}\right) \\
& A_{p} f=\left[\begin{array}{l}
\alpha_{1} f_{1}^{\prime \prime}-F_{1} f_{1}^{\prime}-h_{1} f_{1}+\sqrt{h_{1} h_{2}} f_{2} \\
\alpha_{2} f_{2}^{\prime \prime}+\sqrt{h_{1} h_{2}} f_{1}-\left(h_{2}+h_{3}\right) f_{2}+\sqrt{h_{3} h_{4}} f_{3} \\
\alpha_{3} f_{3}^{\prime \prime}+F_{2} f_{3}^{\prime}+\sqrt{h_{3} h_{4}} f_{2}-\left(h_{4}+h_{5}\right) f_{3}+\sqrt{h_{5} h_{6}} f_{4} \\
\alpha_{4} f_{4}^{\prime \prime}+\sqrt{h_{5} h_{6}} f_{3}-h_{6} f_{4}
\end{array}\right]
\end{aligned}
$$

Ecrivons $A_{p}$ comme la somme d'un opérateur différentiel et d'une application bornée de la façon suivante :

$$
A_{p}=B+C
$$

avec

$$
\begin{aligned}
& D(B)=D\left(A_{d, p}\right), \\
& B f=\left[\begin{array}{c}
\alpha_{1} f_{1}^{\prime \prime}-F_{1} f_{1}^{\prime}+\theta_{1} f_{1} \\
\alpha_{2} f_{2}^{\prime \prime} \\
\alpha_{3} f_{3}^{\prime \prime}+F_{2} f_{3}^{\prime}+\theta_{2} f_{3} \\
\alpha_{4} f_{4}^{\prime \prime}
\end{array}\right], \\
& C f=\left[\begin{array}{cccc}
-\left(h_{1}+\theta_{1}\right) & \sqrt{h_{1} h_{2}} & 0 & 0 \\
\sqrt{h_{1} h_{2}} & -\left(h_{2}+h_{3}\right) & \sqrt{h_{3} h_{4}} & 0 \\
0 & \sqrt{h_{3} h_{4}} & -\left(h_{4}+h_{5}+\theta_{2}\right) & \sqrt{h_{5} h_{6}} \\
0 & 0 & \sqrt{h_{5} h_{6}} & -h_{6}
\end{array}\right] f
\end{aligned}
$$

où $\theta_{1}$ et $\theta_{2}$ sont des constantes positives que nous préciserons par la suite.

Proposition 2 Il existe des constantes $\theta_{1}>0, \theta_{2}>0$ telles que l'opérateur $B$ défini par les équations (7) et (8) soit le générateur d'un $C^{0}$ semi-groupe de contractions sur $X_{p}$.

Preuve. (i). Le domaine $D(B)$ donné par (2) est dense dans $X_{p}=\left(L^{p}(0,1)\right)^{4}$, car il contient l'ensemble $\left(C_{0}^{\infty}(0,1)\right)^{4}$ des fonctions infiniment différentiables et à support compact dans $(0,1)$.

(ii). Prouvons que l'on peut choisir $\theta_{1}>0, \theta_{2}>0$ tels que l'opérateur $B$ soit dissipatif. Notons d'abord que l'on peut écrire

$$
B=\left(B_{1}, B_{2}, B_{3}, B_{4}\right)^{T}
$$

tel que

$$
B f=\left(B_{1} f_{1}, B_{2} f_{2}, B_{3} f_{3}, B_{4} f_{4}\right)^{T}, \quad \forall f=\left(f_{1}, f_{2}, f_{3}, f_{4}\right)^{T} \in X_{p} ;
$$

où les $B_{i}$ sont des opérateurs sur $L^{p}(0,1)$ définis par :

$$
\begin{array}{ll}
D\left(B_{1}\right)=\left\{f_{1} \in W^{2, p} \mid f_{1}(0)=0, f_{1}^{\prime}(1)=0\right\}, & B_{1} f_{1}=\alpha_{1} f_{1}^{\prime \prime}-F_{1} f_{1}^{\prime}+\theta_{1} f_{1} ; \\
D\left(B_{2}\right)=\left\{f_{2} \in W^{2, p} \mid f_{2}^{\prime}(0)=0, f_{2}^{\prime}(1)=0\right\}, & B_{2} f_{2}=\alpha_{2} f_{2}^{\prime \prime} ; \\
D\left(B_{3}\right)=\left\{f_{3} \in W^{2, p} \mid f_{3}^{\prime}(0)=0, f_{3}(1)=0\right\}, & B_{3} f_{3}=\alpha_{3} f_{3}^{\prime \prime}+F_{2} f_{3}^{\prime}+\theta_{2} f_{3} ; \\
D\left(B_{4}\right)=\left\{f_{4} \in W^{2, p} \mid f_{4}^{\prime}(0)=0, f_{4}^{\prime}(1)=0\right\}, & B_{4} f_{4}=\alpha_{4} f_{4}^{\prime \prime} .
\end{array}
$$


Il est clair que la dissipativité de tous les opérateurs $B_{i}$ sur $L^{p}(0,1)$ entrâne celle de l'opérateur $B$ dans $X_{p}$.

Montrons la dissipativité de l'opérateur $B_{1}$. Soit $f_{1} \in D\left(B_{1}\right)$. Sans perte de la généralité on suppose que $\left\|f_{1}\right\|_{p}=1$. Par la Définition 1 et le Lemme 2, on passe par les calculs suivants :

$$
\begin{gathered}
<f_{1}^{*}, B_{1} f_{1}>_{q, p}=\int_{0}^{1}\left|f_{1}\right|^{p-2} f_{1}\left(\alpha_{1} f_{1}^{\prime \prime}-F_{1} f_{1}^{\prime}+\theta_{1} f_{1}\right) d x \\
=-\alpha_{1}(p-1) \int_{0}^{1}\left|f_{1}\right|^{p-2} f_{1}^{\prime 2} d x-\frac{F_{1}}{p}\left|f_{1}\right|^{p}(1)+\theta_{1} \int_{0}^{1}\left|f_{1}\right|^{p} d x .
\end{gathered}
$$

On utilise l'inégalité du type Poincaré suivante:

$$
\int_{0}^{1}|g|^{2} d x \leq \frac{1}{2} \int_{0}^{1}\left|g^{\prime}\right|^{2} d x, \quad \forall g \in W^{1,2}, g(0)=0 .
$$

En posant $g=\left|f_{1}\right|^{\frac{p}{2}}$, on obtient l'inégalité

$$
-\int_{0}^{1}\left|f_{1}\right|^{p-2}\left|f_{1}^{\prime}\right|^{2} d x \leq-\frac{8}{p^{2}} \int_{0}^{1}\left|f_{1}\right|^{p} d x .
$$

En combinant les équations (10) et (11) on obtient

$$
<f_{1}^{*}, B_{1} f_{1}>_{q, p} \leq-\left(\frac{8 \alpha_{1}(p-1)}{p^{2}}-\theta_{1}\right) \int_{0}^{1}\left|f_{1}\right|^{p} d x .
$$

Le choix $\theta_{1}$ tel que

$$
\theta_{1}=\theta_{1}(p)=\frac{8 \alpha_{1}(p-1)}{p^{2}}
$$

donne la dissipativité de l'opérateur $B_{1}$.

Des calculs similaires conduisent à la dissipativité des opérateurs $B_{2}, B_{4}$ et $B_{3}$ en choisissant

$$
\theta_{2}=\theta_{2}(p)=\frac{8 \alpha_{3}(p-1)}{p^{2}} .
$$

(iii). Pour tout $i=1, \ldots, 4$, l'image de l'opérateur $\left(I-B_{i}\right)$ remplit tout l'espace $L^{p}$. On peut le voir en résolvant, pour tout $\varphi \in L^{p}(0,1)$, l'équation différentielle linéaire avec second membre

$$
\left\{\begin{array}{l}
\alpha_{1} u_{1}^{\prime \prime}-F_{1} u_{1}^{\prime}-u_{1}=\varphi \\
u_{1}(0)=0 \\
u_{1}^{\prime}(1)=0
\end{array}\right.
$$

Cette équation admet une unique solution $u_{1} \in W^{2, p}$. Donc $R\left(\left(\theta_{1}+1\right) I-B_{1}\right)$ est surjectif, ce qui entraîne que $R\left(I-B_{1}\right)=L^{p}(0,1)$ (voir Théorème 1.4.5 dans [22]). On conclut que $B$ vérifie la condition d'image et qu'il est le générateur infinitésimal d'un $C^{0}$ semi-groupe de contractions sur $X_{p}, \forall 1<p<\infty$.

Proposition 3. Il existe une constante $\alpha_{p}>0$ telle que l'opérateur $C+\alpha_{p} I, C$ étant défini par l'équation (9), soit dissipatif dans $X_{p}$. 
Preuve. Soit $f \in X_{p}$. On note $g=\left(g_{1}, \ldots, g_{4}\right)^{T}$ tel que

$$
g_{i}=\frac{\left|f_{i}\right|^{p-2} f_{i}}{\left\|f_{i}\right\|_{p}^{p-2}} ; \quad i=1, \ldots, 4 .
$$

Il s'agit de prouver qu'il existe une constante $\alpha>0$ tel que

$$
<g,(C+\alpha I) f>_{q, p} \leq 0, \quad \forall f \in X_{p}
$$

On effectue les calculs successifs suivants :

$$
\begin{aligned}
<g,(C+\alpha I) f>_{q, p}= & \sum_{i=1}^{4} \int_{0}^{1}((C+\alpha I) f)_{i}(x) g_{i}(x) d x \\
= & \int_{0}^{1}\left[\left(-h_{1}-\theta_{1}+\alpha\right) f_{1}+\sqrt{h_{1} h_{2}} f_{2}\right] g_{1} d x \\
& +\int_{0}^{1}\left[\sqrt{h_{1} h_{2}} f_{1}+\left(-h_{2}-h_{3}+\alpha\right) f_{2}+\sqrt{h_{3} h_{4}} f_{3}\right] g_{2} d x \\
& +\int_{0}^{1}\left[\sqrt{h_{3} h_{4}} f_{2}+\left(-h_{4}-h_{5}-\theta_{2}+\alpha\right) f_{3}+\sqrt{h_{5} h_{6}} f_{4}\right] g_{3} d x \\
& +\int_{0}^{1}\left[\sqrt{h_{5} h_{6}} f_{3}+\left(-h_{6}+\alpha\right) f_{4}\right] g_{4} d x
\end{aligned}
$$

On poursuit les calculs en utilisant l'inégalité de Holder,

$$
\begin{aligned}
<g,(C+\alpha I) f>_{q, p} \leq & \left(-h_{1}-\theta_{1}+\alpha\right)\left\|f_{1}\right\|_{p}^{2}+\left(-h_{2}-h_{3}+\alpha\right)\left\|f_{2}\right\|_{p}^{2} \\
& +\left(-h_{4}-h_{5}-\theta_{2}+\alpha\right)\left\|f_{3}\right\|_{p}^{2}+\left(-h_{6}+\alpha\right)\left\|f_{4}\right\|_{p}^{2} \\
& +2 \sqrt{h_{1} h_{2}}\left\|f_{1}\right\|\left\|_{p}\right\| f_{2}\left\|_{p}+2 \sqrt{h_{3} h_{4}}\right\| f_{2}\|\|_{p}\left\|f_{3}\right\|_{p} \\
& +2 \sqrt{h_{5} h_{6}}\left\|f_{3}\right\|_{p}\left\|f_{4}\right\|_{p} .
\end{aligned}
$$

On remarque à ce niveau que si la matrice symétrique $C+\alpha I$ est définie négative, l'opérateur $C+\alpha I$ dans $X_{p}$ est dissipatif. Puisque la matrice $C$ est définie négative, il suffit de prendre $\alpha_{p}$ égal à la plus petite valeur propre de la matrice définie positive $(-C)$ telle que $(C+\alpha I)$ devienne semi-définie négative.

Corollaire 1. L'opérateur $A_{p}$ défini par (5)-(6) est le générateur d'un $C^{0}$ semi-groupe de contractions $S(t)$ sur $X_{p}, 1<p<+\infty$. De plus il existe une constante positive $\alpha_{p}$ telle que

$$
\|S(t)\|_{p} \leq e^{-\alpha_{p} t}, \quad \forall t \geq 0,
$$

c'est-à-dire, le semi-groupe $S(t)$ (ou l'opérateur $A_{p}$ ) est exponentiellement stable sur $X_{p}$.

Preuve. D'après les Propositions 2 et 3 , l'opérateur $B$ est le générateur d'un $C^{0}$ semigroupe de contractions sur $X_{p}$ et l'opérateur $C+\alpha_{p} I$ est borné et dissipatif dans $X_{p}$. En vertu d'un résultat de perturbation (voir Corollaire 3.3, [22, p.82]) on conclut que $A_{p}+\alpha_{p} I=B+\left(C+\alpha_{p} I\right)$ est le générateur d'un $C^{0}$ semi-groupe de contractions sur $X_{p}$. En utilisant $e^{t A_{p}}=e^{t\left(A_{p}+\alpha_{p} I\right)} e^{-t \alpha_{p}}$, on complète la preuve du Corollaire 1. 
Corollaire 2 Pour chaque $p \in(1,+\infty)$, l'opérateur $A_{d, p}$ associé au système d'échangeurs thermiques avec diffusion est le générateur d'un $C^{0}$ semi-groupe (noté $Z(t)$ ) sur $X_{p}$. De plus il existe une constante $M>0$ telle que

$$
\|Z(t)\|_{p} \leq M e^{-\alpha_{p} t}, \quad \forall t \geq 0,
$$

i.e., le semi-groupe $Z(t)$ (ou l'opérateur $A_{d, p}$ ) est exponentiellement stable sur $X_{p}$.

Preuve. On remarque que si $A_{p}$ est le générateur d'un $C^{0}$ semi-groupe $S(t)$, alors $A_{d, p}=$ $T^{-1} A_{p} T$ est aussi le générateur d'un $C^{0}$ semi-groupe $Z(t)$ défini par

$$
Z(t)=T^{-1} S(t) T \text {. }
$$

Donc,

$$
\|Z(t)\| \leq\left\|T^{-1}\right\| \cdot\|T\| \cdot\|S(t)\| .
$$

Le résultat cherché découle du Corollaire 1.

\section{Stabilité du modèle avec diffusion dans $(C[0,1])^{4}$}

\subsection{Générateur infinitésimal}

On considère le système $\left(\Sigma_{d}\right)$ d'échangeurs thermiques dans le Banach $X=(C[0,1])^{4}$ muni de la norme de la convergence uniforme sur chaque composante, plus précisément :

$$
\forall f \in X, \quad\|f\|_{X}=\|f\|_{\infty}=\left[\sum_{i=1}^{4}\left\|f_{i}\right\|_{\infty}^{2}\right]^{\frac{1}{2}} .
$$

L'espace d'état que l'on choisit pour $\left(\Sigma_{d}\right)$ est le sous-espace fermé $X_{\infty}$ dans $X$ défini par

$$
X_{\infty}=\left\{f \in X \mid f_{1}(0)=0, f_{3}(1)=0\right\} \subset X .
$$

L'opérateur associé sur $X_{\infty}$ est défini par

$$
\begin{gathered}
D\left(A_{d, \infty}\right)=\left\{f \in\left(C^{2}[0,1]\right)^{4} \cap X_{\infty} \begin{array}{l}
\alpha_{1} f_{1}^{\prime \prime}(0)-F_{1} f_{1}^{\prime}(0)+h_{1} f_{2}(0)=0 \\
\alpha_{3} f_{3}^{\prime \prime}(1)+F_{2} f_{3}^{\prime}(1)+h_{4} f_{2}(1)+h_{5} f_{4}(1)=0 \\
f_{2}^{\prime}(0)=0, \quad f_{3}^{\prime}(0)=0, \quad f_{4}^{\prime}(0)=0 \\
f_{1}^{\prime}(1)=0, \quad f_{2}^{\prime}(1)=0, \quad f_{4}^{\prime}(1)=0
\end{array}\right\}, \\
A_{d, \infty} f=\left[\begin{array}{l}
\alpha_{1} f_{1}^{\prime \prime}-F_{1} f_{1}^{\prime}+h_{1}\left(f_{2}-f_{1}\right) \\
\alpha_{2} f_{2}^{\prime \prime}+h_{2}\left(f_{1}-f_{2}\right)+h_{3}\left(f_{3}-f_{2}\right) \\
\alpha_{3} f_{3}^{\prime \prime}+F_{2} f_{3}^{\prime}+h_{4}\left(f_{2}-f_{3}\right)+h_{5}\left(f_{4}-f_{3}\right) \\
\alpha_{4} f_{4}^{\prime \prime}+h_{6}\left(f_{3}-f_{4}\right)
\end{array}\right]
\end{gathered}
$$

De même, définissons l'opérateur $A_{\infty}$ sur $X_{\infty}$ par

$$
A_{\infty}=T^{-1} A_{d, \infty} T
$$

avec $T$ la matrice diagonale donnée par (4). On remarque facilement que, pour $1<p<$ $q<+\infty$, on a

$$
D\left(A_{\infty}\right) \subset D\left(A_{q}\right) \subset D\left(A_{p}\right)
$$

et $A_{p}$ prolonge $A_{q}$ qui lui même prolonge $A_{\infty}$. 
Lemme 3. Les propriétés suivantes ont lieu :

(i) L'opérateur $A_{\infty}$ est dissipatif dans $X_{\infty}$.

(ii) L'image $R\left(I-A_{\infty}\right)$ de l'opérateur $I-A_{\infty}$ est $X_{\infty}$.

Preuve. (i). Soit $f \in D\left(A_{\infty}\right)$, alors $f \in D\left(A_{p}\right)$ pour tout $1<p<+\infty$. D'après le Corollaire 1, l'opérateur $A_{p}$ est dissipatif dans $X_{p}$. La Proposition 1 entraîne que

$$
\left\|\left(\lambda I-A_{\infty}\right) f\right\|_{p} \geq \lambda\|f\|_{p}, \quad \forall \lambda>0, \forall p>1 .
$$

En faisant tendre $p$ vers $+\infty$, les quantités $\left\|f_{i}\right\|_{p}$ tendent vers $\left\|f_{i}\right\|_{\infty}$, alors que la relation (20) donne à la limite

$$
\forall \lambda>0,\left\|\left(\lambda I-A_{\infty}\right) f\right\|_{\infty} \geq \lambda\|f\|_{\infty} .
$$

Donc $A_{\infty}$ est dissipatif dans $X$.

(ii). On écrit $A_{\infty}=B_{\infty}+C_{\infty}$, où $B_{\infty}$ et $C_{\infty}$ sont des opérateurs de $X$ définis par

$$
\begin{aligned}
& D\left(B_{\infty}\right)=D\left(A_{\infty}\right), \\
& B_{\infty} f=\left[\begin{array}{l}
\alpha_{1} f_{1}^{\prime \prime}-F_{1} f_{1}^{\prime} \\
\alpha_{2} f_{2}^{\prime \prime} \\
\alpha_{3} f_{3}^{\prime \prime}+F_{2} f_{3}^{\prime} \\
\alpha_{4} f_{4}^{\prime \prime}
\end{array}\right] \\
& C_{\infty} f=\left[\begin{array}{cccc}
-h_{1} & \sqrt{h_{1} h_{2}} & 0 & 0 \\
\sqrt{h_{1} h_{2}} & -\left(h_{2}+h_{3}\right) & \sqrt{h_{3} h_{4}} & 0 \\
0 & \sqrt{h_{3} h_{4}} & -\left(h_{4}+h_{5}\right) & \sqrt{h_{5} h_{6}} \\
0 & 0 & \sqrt{h_{5} h_{6}} & -h_{6}
\end{array}\right] f .
\end{aligned}
$$

On montre que

$$
\rho\left(B_{\infty}\right) \supset(0,+\infty), \quad\left\|R\left(\lambda: B_{\infty}\right)\right\| \leq \lambda^{-1}, \quad \forall \lambda \in(0,+\infty) .
$$

Puisque le domaine $D\left(B_{\infty}\right)$ est dense, par le Théorème de Hill-Yosida [22, p.8], $B_{\infty}$ est le générateur d'un $C_{0}$ semi-groupe sur $X_{\infty}$.

Comme l'opérateur $C_{\infty}$ est borné dans $X_{\infty}$, en utilisant un résultat de perturbation (voir Théorème 1.1 [22, p.76]), on établit que

$$
\rho\left(B_{\infty}+C_{\infty}\right) \supset\left(\left\|C_{\infty}\right\|,+\infty\right) .
$$

En particulier, il existe $\lambda_{0}>0$ tel que $R\left(\lambda_{0} I-A_{\infty}\right)=X_{\infty}$. D'après le Théorème 4.5 [22, p.15], $R\left(I-A_{\infty}\right)=X_{\infty}$.

Nous avons donc démontré le résultat suivant.

Proposition 4 Les opérateurs $A_{\infty}$ et $A_{d, \infty}$ sont tous les deux le générateur d'un $C^{0}$ semi-groupes uniformément bornés sur $X_{\infty}$. 


\subsection{Stabilité exponentielle du modèle avec diffusion dans $X_{\infty}$}

Dans cette section, on montre que le $C^{0}$ semi-groupe engendré par l'opérateur $A_{d, \infty}$ est exponentiellement stable dans $X_{\infty}$ et dans $Y=\left(C^{1}[0,1]\right)^{4}$. Ensuite on propose de caractériser le taux de décroissance exponentielle pour le semi-groupe.

Pour cela nous allons utiliser la théorie des opérateurs sectoriels. D'abord, on montre que l'opérateur $A_{d, \infty}$ est un opérateur sectoriel sur $X_{\infty}$ et sur $Y_{\infty}$. Noter que $Y_{\infty}$ est un sous-espace fermé dans $Y$ défini par

$$
Y_{\infty}=\left\{\begin{array}{l|l}
f \in Y & \begin{array}{l}
f_{1}(0)=f_{1}^{\prime}(1)=0 \\
f_{2}^{\prime}(0)=f_{2}^{\prime}(1)=0 \\
f_{3}^{\prime}(0)=f_{3}(1)=0 \\
f_{4}^{\prime}(0)=f_{4}^{\prime}(1)=0
\end{array}
\end{array}\right\}
$$

Proposition 5. L'opérateur $A_{d, \infty}$ est un opérateur sectoriel sur $X_{\infty}$ et sur $Y_{\infty}$.

Preuve. (1). Par la preuve du Lemme 3, l'opérateur $B_{\infty}$ est le générateur d'un $C^{0}$ semigroupe de contractions sur $X_{\infty}$. Pour montrer que $A_{d, \infty}$ est un opérateur sectoriel, il suffit de prouver que chaque opérateur composant $B_{\infty, i}$ de $B_{\infty}$ est sectoriel sur le sous-espace correspondant de $C[0,1]$. Pour atteindre cet objectif il suffit de prouver qu'il existe des constantes positives $M>0, \omega \in \mathbb{R}$ et $\theta \in] \frac{\pi}{2}, \pi[$ telles que, pour tout $i=1,2,3$ ou 4 , on ait

$$
\begin{aligned}
& \rho\left(B_{\infty, i}\right) \supset S_{\theta, \omega}=\{\lambda \in \mathbb{C},|\operatorname{Arg}(\lambda-\omega)| \leq \theta\} \cup\{\omega\}, \\
& \left\|R\left(\lambda, B_{\infty, i}\right)\right\| \leq \frac{M}{|\lambda-\omega|}, \quad \forall \lambda \in S_{\theta, \omega} .
\end{aligned}
$$

Ceci se réalise par un calcul direct de l'opérateur de résolvante en résolvant l'équation différentielle [27, Chapitre IV]. On en déduit, par le théorème 5.2 [22, p.61], que l'opérateur $B_{\infty}$ est sectoriel sur $X_{\infty}$. Comme $A_{d, \infty}$ est une perturbation bornée de $B_{\infty}$, le résultat découle du Théorème 2.1 dans $[22, \mathrm{p} .80]$.

(2). Pour l'opérateur $A_{d, Y}$ défini sur le sous-espace correspondant dans $Y=\left(C^{1}[0,1]\right)^{4}$, le raisonnement est similaire mais avec un calcul plus élaboré. On commence par prouver que chaque opérateur composant est sectoriel. On laisse au lecteur le soin de la preuve complète pour $A_{d, Y}$ dans $Y_{\infty}$.

Dans la suite, on propose de caractériser les ensembles spectraux pour l'opérateur $A_{d, \infty}$ et pour $A_{d, Y}$.

Proposition 6 Les opérateurs définis sur les espaces de Banach différents $A_{d, Y}, A_{d, \infty}$ et $A_{d, q}$ ont le même ensemble des valeurs propres :

$$
\sigma_{p}\left(A_{d, Y}\right)=\sigma_{p}\left(A_{d, \infty}\right)=\sigma_{p}\left(A_{d, q}\right)
$$

et

$$
\sigma\left(A_{d, Y}\right) \subset \sigma\left(A_{d, \infty}\right) \subset \sigma\left(A_{d, q}\right), \quad \forall 1<q<\infty .
$$


Preuve. Rappelons que

$$
D\left(A_{d, Y}\right)=\left\{f \in Y_{\infty} \cap\left(C^{3}[0,1]\right)^{4} \mid A_{d, \infty} f \in Y_{\infty}\right\} .
$$

1. Montrons d'abord l'égalité des spectres ponctuels $\sigma_{p}\left(A_{d, \infty}\right)=\sigma_{p}\left(A_{d, Y}\right)$. Soit $\lambda \in$ $\sigma_{p}\left(A_{d, Y}\right)$. Alors il existe $f \in D\left(A_{d, Y}\right)$ non nul tel que $\left(\lambda I-A_{d, Y}\right) f=0$. Puisque $A_{d, \infty}$ prolonge $A_{d, Y}$, alors $f \in D\left(A_{d, \infty}\right)$ et $\left(\lambda I-A_{d, \infty}\right) f=0$. Donc $\lambda \in \sigma_{p}\left(A_{d, \infty}\right)$. On en déduit que

$$
\sigma_{p}\left(A_{d, \infty}\right) \supset \sigma_{p}\left(A_{d, Y}\right)
$$

Réciproquement, soit $f \in D\left(A_{d, \infty}\right)$ et soit $\left(\lambda I-A_{d, \infty}\right) f=0$. Par définition, l'équation différentielle ci-dessous admet une solution non triviale $f \in D\left(A_{d, \infty}\right)$ :

$$
\left[\begin{array}{l}
\alpha_{1} f_{1}^{\prime \prime}(x) \\
\alpha_{2} f_{2}^{\prime \prime}(x) \\
\alpha_{3} f_{3}^{\prime \prime}(x) \\
\alpha_{4} f_{4}^{\prime \prime}(x)
\end{array}\right]=\lambda f(x)-\left[\begin{array}{l}
-F_{1} f_{1}^{\prime}(x)+h_{1}\left(f_{2}(x)-f_{1}(x)\right) \\
h_{2}\left(f_{1}-f_{2}\right)+h_{3}\left(f_{3}(x)-f_{2}(x)\right) \\
F_{2} f_{3}^{\prime}(x)+h_{4}\left(f_{2}(x)-f_{3}(x)\right)+h_{5}\left(f_{4}(x)-f_{3}(x)\right) \\
h_{6}\left(f_{3}(x)-f_{4}(x)\right)
\end{array}\right]
$$

Par conséquent, la solution $f \in\left(C^{3}[0,1]\right)^{4}$ et $A_{d, \infty} f \in Y_{\infty}$. Donc $f \in D\left(A_{d, Y}\right)$. On vient de montrer que

$$
\sigma_{p}\left(A_{d, \infty}\right) \subset \sigma_{p}\left(A_{d, Y}\right) .
$$

On a ainsi prouvé l'égalité des spectres ponctuels.

(2). Le raisonnement similaire à celui du point (1) permet d'établir que

$$
\sigma_{p}\left(A_{d, \infty}\right)=\sigma_{p}\left(A_{d, q}\right)
$$

(3). Il est évident que $\rho\left(A_{d, Y}\right) \supset \rho\left(A_{d, \infty}\right) \supset \rho\left(A_{d, q}\right)$, ce qui permet d'écrire

$$
\sigma\left(A_{d, Y}\right) \subset \sigma\left(A_{d, \infty}\right) \subset \sigma\left(A_{d, q}\right) .
$$

Nous sommes en mesure d'établir le théorème de stabilité dans le cadre des espaces de Banach $X_{\infty}$ et $Y_{\infty}$.

Théorème 1. Les taux de décroissance exponentielle des semi-groupes engendrés par $A_{d, \infty}$ et $A_{d, Y}$, respectivement, sur $X_{\infty}$ et $Y_{\infty}$, sont identiques :

$$
\omega_{0}\left(A_{d, \infty}\right)=\omega_{0}\left(A_{d, Y}\right)=S\left(A_{d, \infty}\right)=\sup _{\lambda \in \sigma_{p}\left(A_{d, \infty}\right)} \Re e(\lambda)<0 .
$$

Par conséquent, le semi-groupe associé au système d'échangeurs thermiques avec diffusion est exponentiellement stable sur $X_{\infty}$ et $Y_{\infty}$.

Preuve. D'après le Corollaire 2, l'opérateur $A_{d, 2}$ est le générateur d'un $C^{0}$ semi-groupe exponentiellement stable sur $X_{2}=\left(L^{2}(0,1)\right)^{4}$. De plus, on montre que le semi-groupe engendré est analytique et que $A_{d, 2}$ est à résolvantes compactes, donc $\sigma_{p}\left(A_{d, 2}\right)=\sigma\left(A_{d, 2}\right)$. Donc, on a

$$
\omega_{0}\left(A_{d, 2}\right)=S\left(A_{d, 2}\right)=\sup _{\lambda \in \sigma_{p}\left(A_{d, 2}\right)} \Re e(\lambda)
$$


Puisque $A_{d, \infty}$ et $A_{d, Y}$ sont sectoriels sur $X_{\infty}$ et $Y_{\infty}$, respectivement, alors on a aussi

$$
\omega_{0}\left(A_{d, \infty}\right)=S\left(A_{d, \infty}\right), \quad \omega_{0}\left(A_{d, Y}\right)=S\left(A_{d, Y}\right) .
$$

A l'aide de la Proposition 6 et du fait que $A_{d, \infty}$ et $A_{d, Y}$ sont à résolvantes compactes, nous obtenons de (26)-(27):

$$
\omega_{0}\left(A_{d, Y}\right)=\omega_{0}\left(A_{d, \infty}\right)=\omega_{0}\left(A_{d, 2}\right)<0
$$

D'où le résultat cherché.

\section{Stabilité du modèle sans diffusion dans $(C[0,1])^{4}$ et $\left(L^{p}(0,1)\right)^{4}$}

On considère le système $\left(\Sigma_{c}\right)$ d'échangeurs thermiques sans diffusion (i.e., avec convection). On note par $A_{c, \infty}$ et $A_{c, p}$, respectivement, les opérateurs sur $X=(C[0,1])^{4}$ et $Y_{p}=\left(L^{p}(0,1)\right)^{4}$ associés au système $\left(\Sigma_{c}\right)$. Ils sont définis par

$$
\begin{aligned}
& D\left(A_{c, \infty}\right)=\left\{f \in\left(C^{1}(0,1)\right)^{4} \mid f_{1}(0)=0, f_{3}(1)=0\right\} \\
& D\left(A_{c, p}\right)=\left\{f \in\left(W^{1, p}(0,1)\right)^{4} \mid f_{1}(0)=0, f_{3}(1)=0\right\} \\
& A_{c, p} f=A_{c, \infty} f=\left[\begin{array}{l}
-F_{1} f_{1}^{\prime}+h_{1}\left(f_{2}-f_{1}\right) \\
h_{2}\left(f_{1}-f_{2}\right)+h_{3}\left(f_{3}-f_{2}\right) \\
F_{2} f_{3}^{\prime}+h_{4}\left(f_{2}-f_{3}\right)+h_{5}\left(f_{4}-f_{3}\right) \\
h_{6}\left(f_{3}-f_{4}\right)
\end{array}\right] .
\end{aligned}
$$

Rappelons que

$$
W^{1, p}(0,1)=\left\{f_{1} \in L^{p}(0,1) \mid f_{1}^{\prime} \in L^{p}(0,1)\right\}
$$

On effectue la même transformation que précédemment sur les opérateurs:

$$
E_{c}=T A_{c, \infty} T^{-1}, \quad E_{p}=T A_{c, p} T^{-1}
$$

où $T \in \mathbb{R}^{4 \times 4}$ est la matrice diagonale donnée par (4).

On décompose $E_{p}$ comme la somme d'un opérateur différentiel et d'une application matricielle bornée:

$$
E_{p}=G+\Lambda
$$


avec

$$
\begin{aligned}
& D(G)=D\left(A_{c, p}\right) \\
& G f=\left[\begin{array}{l}
-F_{1} f_{1}^{\prime} \\
0 \\
F_{2} f_{3}^{\prime} \\
0
\end{array}\right] \\
& \Lambda=\left[\begin{array}{cccc}
-h_{1} & \sqrt{h_{1} h_{2}} & 0 & 0 \\
\sqrt{h_{1} h_{2}} & -\left(h_{2}+h_{3}\right) & \sqrt{h_{3} h_{4}} & 0 \\
0 & \sqrt{h_{3} h_{4}} & -\left(h_{4}+h_{5}\right. & \sqrt{h_{5} h_{6}} \\
0 & 0 & \sqrt{h_{5} h_{6}} & -h_{6}
\end{array}\right]
\end{aligned}
$$

Nous pouvons démontrer le résultat suivant.

Théorème 2 (i) L'opérateur $E_{p}$ est le générateur d'un $C^{0}$ semi-groupe de contractions sur $Y_{p}$.

(ii) L'opérateur $A_{c, p}$ est le générateur d'un $C^{0}$ semi-groupe $S_{p}(t)$ borné sur $Y_{p}$.

(iii) La trace de l'opérateur $A_{c, \infty}$ sur l'espace $X_{0}=\left\{f \in X \mid f_{1}(0)=f_{3}(0)=0\right\}$ est le générateur d'un $C^{0}$ semi-groupe $W_{0}(t)$ borné sur $X_{0}$.

Preuve. Pour prouver (i), on montre d'abord que l'opérateur $G$ précédent engendre un $C^{0}$ semi-groupe de contractions sur $Y_{p}$. Soit $f \in Y_{p}$, notons $g=\left(g_{1}, \ldots, g_{4}\right)^{T}$ tel que

$$
\begin{aligned}
g_{i}=\frac{\left|f_{i}\right|^{p-2} f_{i}}{\left\|f_{i}\right\|_{p}^{p-2}} ; \quad i=1, \ldots, 4 \\
<g, G f>_{Y_{q}, Y_{p}}=\sum_{i=1}^{4} \int_{0}^{1}(G f)_{i}(x) g_{i}(x) d x \\
=-\frac{F_{1}}{\left\|f_{1}\right\|_{p}^{p-2}} \int_{0}^{1}\left|f_{1}(x)\right|^{p-2} f_{1}(x) f_{1}^{\prime}(x) d x \\
+\frac{F_{2}}{\left\|f_{3}\right\| p_{p}^{p-2} \int_{0}^{1}\left|f_{3}(x)\right|^{p-2} f_{3}(x) f_{3}^{\prime}(x) d x} \\
=-\frac{1}{p} \frac{F_{1}}{\left\|f_{1}\right\|_{p}^{p-2}}\left[\left|f_{1}(x)\right|^{p}\right]_{x=0}^{x=1}+\frac{1}{p} \frac{F_{2}}{\left\|f_{3}\right\|_{p}^{p-2}}\left[\left|f_{3}(x)\right|^{p}\right]_{x=0}^{x=1} \\
=-\frac{1}{p} \frac{F_{1}}{\left\|f_{1}\right\|_{p}^{p-2}}\left|f_{1}(1)\right|^{p}-\frac{1}{p} \frac{F_{2}}{\left\|f_{3}\right\|_{p}^{p-2}}\left|f_{3}(0)\right|^{p} \leq 0 .
\end{aligned}
$$

D'où la dissipativité de l'opérateur $G$ sur $Y_{p}$. On montre que $G$ vérifie la condition d'image en utilisant la même démarche qu'à la preuve de la Proposition 2.

Donc $G$ engendre un $C^{0}$ semi-groupe de contractions sur $Y_{p}$, puisque $Y_{p}$ est un Banach réflexif.

On voit clairement que la matrice $\Lambda$ est dissipative dans $Y_{p}$ (voir preuve de la Proposition 3). On déduit, par un résultat de perturbations, que $A_{c, p}=G+\Lambda$ est le générateur d'un 
$C^{0}$ semi-groupe de contractions sur $Y_{p}$.

On établit (ii) exactement comme à la preuve du Corollaire 2.

Enfin pour (iii), on établit que l'opérateur $E_{c}$ est dissipatif et vérifie la condition d'image sur $X$ par le même raisonnement qu'au Lemme 3. Ensuite, par le même raisonnement qu'à la Proposition 4 on montre que la trace de $E_{c}$ sur $X_{0}$ est dissipative, vérifie la condition d'image et est de domaine dense dans $X_{0}$. D'où le résultat voulu.

Pour la suite, donnons la définition suivante.

Définition 2 Le $C^{0}$ semi-groupe sur $Y_{p}$ (resp. $X_{0}$ ) engendrépar l'opérateur $A_{c, p}$ (resp. trace de $A_{c, \infty}$ sur $X_{0}$ ) est appelé le semi-groupe associé au modèle de convection sur $Y_{p}$ (resp. $\left.X_{0}\right)$.

Pour étudier la stabilité du semi-groupe engendré par $A_{c, \infty} \operatorname{sur} X$ (ou plutôt sa trace sur $X_{0}$ ), nous allons passer à la transformation de l'opérateur.

On effectue dans le système $\left(\Sigma_{c}\right)$ le changement de fonction inconnue de la façon suivante:

$$
u_{i}=v_{i} e^{\theta x}, \quad i=1,2,3,4
$$

où $\theta$ est un réel arbitraire. Alors $v=\left(v_{1}, v_{2}, v_{3}, v_{4}\right)^{T}$ est la solution du système suivant :

$$
\left(\widetilde{\Sigma}_{c}\right):\left\{\begin{array}{l}
\partial_{t} v_{1}=-F_{1} \partial_{x} v_{1}-\theta F_{1} v_{1}+h_{1}\left(v_{2}-v_{1}\right), \quad x \in(0,1), \quad t>0 \\
\partial_{t} v_{2}=h_{2}\left(v_{1}-v_{2}\right)+h_{3}\left(v_{3}-v_{2}\right) \\
\partial_{t} v_{3}=F_{2} \partial_{x} v_{3}+\theta F_{2} v_{3}+h_{4}\left(v_{2}-v_{3}\right)+h_{5}\left(v_{4}-v_{3}\right) \\
\partial_{t} v_{4}=h_{6}\left(v_{3}-v_{4}\right) \\
v_{1}(0, t)=0, \quad v_{3}(1, t)=0 \\
v(x, 0)=v^{0}(x), \quad x \in(0,1)
\end{array}\right.
$$

On introduit les opérateurs $A_{c, \theta}$ et $A_{p, \theta}$ sur $X_{0}$ et $Y_{p}$ respectivement;

$$
\begin{aligned}
& D\left(A_{c, \theta}\right)=\left\{f \in\left(C^{1}(0,1)\right)^{4} \mid f_{1}(0)=0, f_{3}(1)=0\right\} \\
& D\left(A_{p, \theta}\right)=\left\{f \in\left(W^{1, p}(0,1)\right)^{4} \mid f_{1}(0)=0, f_{3}(1)=0\right\} \\
& A_{p, \theta} f=A_{c, \theta} f=\left[\begin{array}{l}
-F_{1} f_{1}^{\prime}-\theta F_{1} f_{1}+h_{1}\left(f_{2}-f_{1}\right) \\
h_{2}\left(f_{1}-f_{2}\right)+h_{3}\left(f_{3}-f_{2}\right) \\
F_{2} f_{3}^{\prime}+\theta F_{2} f_{3}+h_{4}\left(f_{2}-f_{3}\right)+h_{5}\left(f_{4}-f_{3}\right) \\
h_{6}\left(f_{3}-f_{4}\right)
\end{array}\right] .
\end{aligned}
$$

On effectue la même transformation que précédemment :

$$
E_{c, \theta}=T^{-1} A_{c, \theta} T, \quad E_{p, \theta}=T^{-1} A_{p, \theta} T .
$$


On fait la décomposition de $E_{p, \theta}$ comme la somme d'un opérateur différentiel et d'une application matricielle bornée : $E_{p, \theta}=G+\Lambda_{\theta}$ avec

$$
\begin{aligned}
& D(G)=D\left(A_{p, \theta}\right) \\
& G f=\left[\begin{array}{l}
-F_{1} f_{1}^{\prime} \\
0 \\
F_{2} f_{3}^{\prime} \\
0
\end{array}\right] \\
& \Lambda_{\theta}=\left[\begin{array}{cccc}
-\left(h_{1}+\theta F_{1}\right) & \sqrt{h_{1} h_{2}} & 0 & 0 \\
\sqrt{h_{1} h_{2}} & -\left(h_{2}+h_{3}\right) & \sqrt{h_{3} h_{4}} & 0 \\
0 & \sqrt{h_{3} h_{4}} & -\left(h_{4}+h_{5}-\theta F_{2}\right) & \sqrt{h_{5} h_{6}} \\
0 & 0 & \sqrt{h_{5} h_{6}} & -h_{6}
\end{array}\right]
\end{aligned}
$$

On établit le résultat important suivant.

Proposition 7. Il existe des constantes $\theta>0$ et $\mu>0$ indépendantes de $p$ telles que l'opérateur $E_{p, \theta}+\mu I$ soit le générateur d'un semi-groupe de contractions sur $Y_{p}$.

Pour prouver cette proposition, nous aurons besoin des lemmes qui suivent.

Lemme 4 Il existe des constantes $\theta>0$ et $\mu>0$ indépendantes de $p$ telles que l'opérateur $\Lambda_{\theta}+\mu I$ soit dissipatif dans $Y_{p}$.

Preuve. Soit $f \in D(G)$, notons $g=\left(g_{1}, \ldots, g_{4}\right)^{T}$ tel que

$$
g_{i}=\frac{\left|f_{i}\right|^{p-2} f_{i}}{\left\|f_{i}\right\|_{p}^{p-2}} ; \quad i=1, \ldots, 4 .
$$

On effectue les mêmes calculs qu'à la preuve de la Proposition 3. A l'aide des inégalités de Hölder et de Cauchy, on peut écrire

$$
\begin{aligned}
& <g,\left(\Lambda_{\theta}+\mu I\right) f>_{Y_{q}, Y_{p}}= \\
& =\left\langle\left[\begin{array}{l}
g_{1} \\
g_{2} \\
g_{3} \\
g_{4}
\end{array}\right],\left[\begin{array}{c}
{\left[-h_{1}-\theta F_{1}+\mu\right] f_{1}+\sqrt{h_{1} h_{2}} f_{2}} \\
\sqrt{h_{1} h_{2}} f_{1}+\left[-h_{2}-h_{3}+\mu\right] f_{2}+\sqrt{h_{3} h_{4}} f_{3} \\
\sqrt{h_{3} h_{4}} f_{2}+\left[-h_{4}-h_{5}+\theta F_{2}+\mu\right] f_{3}+\sqrt{h_{5} h_{6}} f_{4} \\
\sqrt{h_{5} h_{6}} f_{3}-h_{6} f_{4}+\mu f_{4}
\end{array}\right]\right\rangle_{q, p} \\
& =<g_{1},\left(-h_{1}-\theta F_{1}+\mu\right) f_{1}+\sqrt{h_{1} h_{2}} f_{2}>_{q, p} \\
& +<g_{2}, \sqrt{h_{1} h_{2}} f_{1}+\left(-h_{2}-h_{3}+\mu\right) f_{2}+\sqrt{h_{3} h_{4}} f_{3}>_{q, p} \\
& +<g_{3}, \sqrt{h_{3} h_{4}} f_{2}+\left(-h_{4}-h_{5}+\theta F_{2}+\mu\right) f_{3}+\sqrt{h_{5} h_{6}} f_{4}>_{q, p} \\
& +<g_{4}, \sqrt{h_{5} h_{6}} f_{3}+\left(-h_{6}+\mu\right) f_{4}>_{q, p} \leq\left[\begin{array}{c}
\left\|f_{1}\right\|_{p} \\
\left\|f_{2}\right\|_{p} \\
\left\|f_{3}\right\|_{p} \\
\left\|f_{4}\right\|_{p}
\end{array}\right]^{\top}\left(\widetilde{\Lambda}_{\theta}+\mu I\right)\left[\begin{array}{c}
\left\|f_{1}\right\|_{p} \\
\left\|f_{2}\right\|_{p} \\
\left\|f_{3}\right\|_{p} \\
\left\|f_{4}\right\|_{p}
\end{array}\right] \text {, }
\end{aligned}
$$


où

$$
\widetilde{\Lambda}_{\theta}=\left[\begin{array}{cccc}
-\left(h_{1}+\theta F_{1}\right) & \sqrt{h_{1} h_{2}} & 0 & 0 \\
\sqrt{h_{1} h_{2}} & -\left(h_{2}+h_{3}\right) & \sqrt{h_{3} h_{4}} & 0 \\
0 & \sqrt{h_{3} h_{4}} & -\left(h_{4}+h_{5}-\theta F_{2}\right) & \sqrt{h_{5} h_{6}} \\
0 & 0 & \sqrt{h_{5} h_{6}} & -h_{6}
\end{array}\right] .
$$

On peut trouver une constante $\theta>0$ telle que la matrice $\left(-\widetilde{\Lambda}_{\theta}\right)$ soit définie positive, il suffit de prendre sa plus petite valeur propre pour $\mu$ :

$$
\mu=\min _{i=1, \ldots, 4} \sigma_{i}\left(-\widetilde{\Lambda}_{\theta}\right)
$$

Cela permet d'obtenir l'inégalité suivante :

$$
<g,\left(\Lambda_{\theta}+\mu I\right) f>_{Y_{q}, Y_{p}} \leq-\left[\begin{array}{c}
\left\|f_{1}\right\|_{p} \\
\left\|f_{2}\right\|_{p} \\
\left\|f_{3}\right\|_{p} \\
\left\|f_{4}\right\|_{p}
\end{array}\right]^{\top}\left(-\widetilde{\Lambda}_{\theta}-\mu I\right)\left[\begin{array}{c}
\left\|f_{1}\right\|_{p} \\
\left\|f_{2}\right\|_{p} \\
\left\|f_{3}\right\|_{p} \\
\left\|f_{4}\right\|_{p}
\end{array}\right] \leq 0 .
$$

On complète ainsi la preuve du Lemme 4.

Remarque 1. On peut aussi choisir une constante $\theta<0$ telle que $\left(-\widetilde{\Lambda}_{\theta}\right)$ soit définie positive. Par conséquent il existe des constantes $\theta<0$ et $\mu>0$ telles que $\left(\Lambda_{\theta}+\mu I\right)$ soit dissipatif.

Lemme 5. L'opérateur $G$ défini par (38)-(39) est le générateur d'un $C^{0}$ semi-groupe de contractions sur $Y_{p}$.

Preuve. La preuve de ce lemme est donnée au début de la preuve du Théorème 2.

Preuve de Proposition 7 : D'après le Lemme 4, il existe $\theta>0$ et $\mu>0$ telles que $\left(\Lambda_{\theta}+\mu I\right)$ soit dissipatif. Par le Lemme 5, l'opérateur $E_{p, \theta}+\mu I=G+\Lambda_{\theta}+\mu I$ est aussi dissipatif et donc le générateur d'un $C^{0}$ semi-groupe de contractions sur $Y_{p}$.

Maintenant, nous énonçons le résultat de stabilisation.

Théorème 3. (i) Pour tout $1<p<+\infty$, le semi-groupe $W_{p}(t)$ sur $Y_{p}=\left(L^{p}(0,1)\right)^{4}$ associé au modèle de diffusion est exponentiellement stable.

(ii) Le semi-groupe $W_{0}(t)$ sur $X_{0}$ associé au modèle de convection est exponentiellement stable.

Preuve. (i) La Proposition 7 assure l'existence de $\mu>0$ et $\theta \in \mathbb{R}$ tels que l'opérateur $E_{p, \theta}+\mu I$ soit générateur d'un $C^{0}$ semi-groupe de contractions sur $Y_{p}$. En notant par $T_{p, \theta}(t)$ le $C^{0}$ semi-groupe sur $Y_{p}$ engendré par $E_{p, \theta}$, on établit que

$$
\forall w \in Y_{p}, \quad W_{p}(t) w(x)=e^{\theta x} T_{p, \theta}(t)\left(e^{-\theta \cdot} w\right)(x) .
$$

Le semi-groupe $T_{p, \theta}$ est exponentiellement stable :

$$
T_{p, \theta}=e^{-\mu t} e^{\left(E_{p, \theta}+\mu I\right) t} .
$$


Par conséquent, il existe une constante $M>0$ dépendant de $\theta$ telle que

$$
\left\|W_{p}(t)\right\| \leq M e^{-\mu t}, \quad \forall t \geq 0 .
$$

(ii) En vertu de la Proposition 7, il existe $\mu>0$ et $\theta \in \mathbb{R}$ (indépendants de p) tels que l'opérateur sur $Y_{p} E_{p, \theta}+\mu I$ soit dissipatif. En passant en limite, on prouve que l'opérateur $E_{c, \theta}+\mu I$ est dissipatif (similaire à la preuve de la Proposition 2 (iii)) sur $X_{0}$ et vérifie la condition d'image. Par conséquent, l'opérateur $E_{c, \theta}$ sur $X_{0}$ engendre un semi-groupe uniformément borné sur $X_{0}$ que nous notons $T_{\theta}(t)$. En notant $W_{0}(t)$ le semi-groupe sur $X_{0}$ engendré par $E_{c}$, on établit une relation similaire à (42) entre $W_{0}(t)$ et $T_{\theta}(t)$. Par conséquent, il existe une constante $M>0$ dépendant de $\theta$ telle que

$$
\left\|W_{0}(t)\right\| \leq M e^{-\mu t}, \quad \forall t \geq 0 .
$$

Ainsi on achève la preuve du Théorème 3.

Enfin nous indiquons que le semi-groupe associé au modèle de convection n'est pas analytique.

Proposition 8 le semi-groupe ( $\operatorname{sur} Y_{p}$ ou $X_{0}$ ) associé au modèle de convection n'est pas analytique.

Preuve de Proposition 8 : Il suffit de prouver que l'opérateur de transport $\mathcal{T}$ sur $C[0,1]$ suivant

$$
D(\mathcal{T})=\left\{f \in C^{1}[0,1]: f(0)=0\right\} ; \quad \mathcal{T} f=-f_{x}
$$

n'est pas sectoriel. En effet l'opérateur de résolvante s'écrit

$$
R(\lambda, \mathcal{T}) f(x)=u(x)=\int_{0}^{x} e^{\lambda(\xi-x)} f(\xi) d \xi, \quad \forall f \in X, \forall \lambda \in \mathbb{C} .
$$

Supposons que $\mathcal{T}$ est sectoriel. Puisque son semi-groupe engendré est borné, son ensemble de résolvante $\rho(\mathcal{T})$ contient l'ensemble $\Sigma$ des $\lambda=n+i m$ tels que $n<0$ et $\left|\frac{n}{m}\right|$ soit suffisamment petit. De plus, il existe une constante $M>0$ telle que

$$
|\lambda|\|R(\lambda, \mathcal{T}) f\| \leq M\|f\|, \quad \forall \lambda \in \Sigma, \forall f \in C[0,1] .
$$

Soit $f \equiv 1$ et soit $\lambda=n+i m$. La relation (43) entraîne

$$
|u(x)|=\left|\frac{1-e^{-(n+i m) x}}{n+i m}\right| \geq \frac{e^{-n x}-1}{|\lambda|} .
$$

Cela permet d'écrire l'inégalité suivante pour $n$ grand :

$$
\begin{gathered}
\|u\|_{p} \geq\left(\int_{1 / 2}^{1}|u(x)|^{p} d x\right)^{1 / p} \geq \frac{1}{|\lambda|}\left(\int_{1 / 2}^{1}\left(e^{-n x}-1\right)^{p} d x\right)^{1 / p} \\
\geq \frac{1}{2|\lambda|}\left(\int_{1 / 2}^{1} e^{-p n x} d x\right)^{1 / p}=\frac{1}{2|\lambda|}\left(\frac{e^{-n p}-e^{-n p / 2}}{-n p}\right)^{1 / p} .
\end{gathered}
$$

Comme la fonction exponentielle emporte sur le polynôme, à partir de (45) on obtient

$$
|\lambda|\|R(\lambda, \mathcal{T}) f\|=|\lambda| \cdot\|u\| \rightarrow+\infty
$$


lorsque $n \rightarrow-\infty$. Ceci est en contradiction avec (44). Donc le semi-groupe n'est pas analytique dans $Y_{p}$.

Maintenant, soit $f(x)=e^{x}-1$. Soit $\lambda \in \Sigma$. Par (43), on trouve $u(x)$ :

$$
u(x)=\frac{e^{x}}{\lambda+1}-\frac{1}{\lambda}+\frac{e^{-\lambda x}}{\lambda(\lambda+1)} .
$$

Alors,

$$
|\lambda|\|R(\lambda, \mathcal{T}) f\|_{\infty}=|\lambda|\|u\|_{\infty} \geq \frac{e^{-n}}{|\lambda+1|}-1-\left|\frac{\lambda e}{\lambda+1}\right| .
$$

La fonction exponentielle emportant sur le polynôme, le membre de l'extrême droite de (46) tend vers $+\infty$ quand $n \rightarrow-\infty$. Or, cela est en contradiction avec (44). Par conséquent le semi-groupe n'est pas analytique dans $X_{0}$, non plus.

\section{References}

[1] S. Agmon, On the eigenfunctions and the eigenvalues of general elliptic boundary value problem, Comm. Pure Appl. Math. 15, 119-147, 1962.

[2] Ph. Bénilan, H. Touré, Sur l'équation générale $u_{t}=a\left(., u, \varphi(., u)_{x}\right)_{x}$ dans $L^{1}, I I$, Etude du problème d'évolution, Ann. Inst. Henri Poincaré, Vol. 12, nº, p. 727-761, 1995.

[3] H. Bounit, H. Hammouri, J. Sau, Regulation of an irrigation canal system through the semigroup approach, Proceedings of the International Workshop Regulation of Irrigation Canals : Sates of the Art of Research and Applications, 261-276, Morocco, 1997.

[4] M. Bouyssel, Integrale de Lebesgue, mesure et integration; Cépadues-Editions, 1996.

[5] H. Brézis, Opérateurs maximaux monotones et semi-groupes de contractions dans les espaces de Hilbert, North Holland, Amsterdam, 1973.

[6] H. Brézis, Analyse fonctionnelle: théorie et applications, Masson, Paris, 1983.

[7] N. Chafee, Asymptotic behaviour for solutions of a one-dimensional parabolic equation with homogeneous Neumann boundary conditions, J. Differential Equations 18, 111-134, 1975.

[8] J.M. Coron, On the null asymptotic stabilization of the two-dimansional incompressible Euler equations in a simply connected domain, Siam Journal on Control and Optimization, vol.37, 1874-1896, 1999.

[9] L.C. Evans, Partial differential equations, American Mathematical Society, Providence, RI, 1998.

[10] A. Friedman, Partial differential equations of parabolic type, Prentice-Hall, 1963.

D. Gilbarg, N. Trudinger, Elliptic partial differential equations of second order, Springer, 1977.

[11] A. Haraux, Systèmes dynamiques dissipatifs et applications, Masson, Paris, 1991.

[12] F. Huang, Characteristic conditions for exponential stability of linear dynamical systems in Hilbert spaces, Ann. Diff. Eqns 1:45-53, 1985.

[13] V. Komornik, Exact controllability and stabilization: the multiplier method, Masson, Paris, 1994.

[14] O. Ladyzhenskaya, N. Uralceva, Linear and quasilinear elliptic equations, Acad. Press, 1968 (Traduction française Dunod).

[15] O. Ladyzhenskaya, V. Solonnikov, N. Uralceva, Linear and quasilinear equations of parabolic type, Amer. Math. society, 1968. 
[16] J. Liéto, Le génie chimique à l'usage des chimistes, Lavoisier TEC \& DOC, 1998.

[17] J.L. Lions, Contrôlabilité exacte perturbations et stabilisation de systèmes distribués, Tome 1: Contrôlabilité exacte, Masson, 1988.

[18] C. Lobry, Bases mathématiques de la théorie des systèmes asservis non linéaires, Analyse Appliquée et Informatique, Université Bordeaux 1, 1976.

[19] H. Matano, Asymptotic behaviour and stability of solutions of semilinear diffusion equations, Publ. RIMS Kyoto University, 15, 401-454, 1979.

[20] H. Matano, Convergence of solutions of one-dimensional semilinear parabolic equations, $J$. Math. Kyoto Univ., vol. 18-2, 221-227, 1978.

[21] U. Nieken, G. Kolios, G. Eigenberger, Limiting cases and approximate solutions for a fixedbed reactors with periodic flow reversal, AiChe, vol.41, no.8, August 1995.

[22] A. Pazy, Semigroups of Linear Operators and Applications to Partial Differential Equations, Springer-Verlag, New York, 1983.

[23] M.H. Protter, H.F. Weinberger, Maximum principles in differential equations, SpringerVerlag, New York, 1984.

[24] J. Rauch, M. Taylor, Exponential decay of solutions to hyperbolic equations in bounded domain, Indiana university Mathematics journal, 24, 79-86, 1974.

[25] M. Renardy, On the linear stability of hyperbolic PDE's and viscoelastic flows, Z. Angew Math. Phys. (ZAMP), vol.45, 854-865, 1994.

[26] F. Simondon, H. Touré, A Lyapunov functional and long-time behaviour for a degenerate parabolic problem, Advances in Mathematical sciences and applications, Gakkôtosho, Tokyo, vol.6, no.1 , 243-266, 1996.

[27] A. Tchousso, Étude de la stabilité asymptotique de quelques modèles de transfert de chaleur, Thèse doctorale, 141 pages, Université Claude Bernard Lyon 1, 2004.

[28] A. Tchousso, C.Z. Xu, Exponential stability of symmetric hyperbolic systems using Lyapunov functionals, to appear in the Proceedings of Methods and Models in Automation and Robotics, Miedzydroje, Poland, September 2004.

[29] H. Touré, Etude des équations générales $u_{t}-\varphi(u)_{x x}+f(u)_{x}=v$ par la théorie des semi-groupes non linéaires dans $L^{1}$, Thèse de $3^{\text {eme }}$ cycle, Université de Franche Comté, 1982.

[30] D. Vortmeyer, R.J. Schaefer, Equivalence of one- and two-phase models for heat transfer processes in packed beds: one dimensional theory, chemical Engineering Science, vol.29, pp. 485-491, 1974.

[31] C.Z. Xu, Commande des systèmes à paramètres distribués, Mémoire de l'Habilitation à Diriger la Recherche, 58 pages, Université de Metz, 1997.

[32] C.Z. Xu, G. Sallet, Exponential stability and transfer functions of processes governed by symmetric hyperbolic systems, ESAIM : Control, Optimization and Calculus of Variations, vol.7, 421-442, June 2002 (URL:http://www.emath.fr/cocv/).

[33] C.Z. Xu, G. Sallet, Proportional and integral regulation of irrigation canal systems governed by the St Venant equation, The proceedings of the IFAC world congress 1999, 147-152, Beijing, P.R. China, 1999.

[34] C.Z. Xu, J.P. Gauthier, Analyse et commande d'un échangeur thermique à contre-courant, R.A.I.R.O. - APII, vol.25, 377-396, 1991.

[35] T.I. Zelenyak Stabilisation of solutions of boundary value problems for a second order parabolic equation with one space variable, Differentsial'nye Uralvneniya, 4, 34-35, 1968 (Translated as Diff. Eq., 4, 17-22, 1968). 